\title{
Lax Prophylaxis: Vaccinating the Inflammatory Bowel Disease Patient
}

\author{
Grigoriy E. Gurvits ${ }^{1}$
}

Published online: 10 November 2017

(C) Springer Science+Business Media, LLC, part of Springer Nature 2017

Inflammatory bowel disease (IBD) is a chronic medical condition affecting an estimated 1.5 million Americans, with 70,000 new cases added annually-a number that has been on the rise since the late twentieth century. The healthcare burden of IBD is largely reflected in the nearly 92,000 hospitalizations, $1,300,000$ physician visits and 120,000 annual disabilities resulting in a $\$ 6.3$ billion cost annually [1]. Disease progression is significant; traditionally, up to three quarters of Crohn's disease (CD) and one quarter of ulcerative colitis (UC) patients require surgery in their lifetime [1]. Similarly, IBD patients have an $80 \%$ estimated lifetime probability for steroid therapy, $40 \%$ for immunomodulatory therapy, and $20 \%$ biologic therapy resulting in high likelihood of immunosuppression [2]. The rapid growth of healthcare research in the twenty-first century has led to an ever-increasing armamentarium of available remedies, of which infliximab and adalimumab have become the commonest prescribed medications for patients with moderate-severe IBD. Nevertheless, as effective as biologics are in achieving and maintaining clinical and histologic remission as well as improving patients' quality-of-life, their use places recipients at a higher risk of infections. In this respect, the paper by Dr. Pham and colleagues [3] published in current issue of Digestive Diseases and Sciences yet again highlights an important issue of precautionary practices in the IBD population, examining the rates and predictors of

Grigoriy E. Gurvits

g_gurvits@hotmail.com

1 Division of Gastroenterology, Department of Medicine, New York University School of Medicine, Langone Medical Center, 555 Madison Avenue, 3 Fl, New York, NY 10022, USA vaccinations among IBD patients initiated on infliximab and adalimumab therapy in an integrated West Coast healthcare system.

The idea of vaccinating immunocompromised patients is not new. Indeed, the annual vaccination schedule released by the US Centers for Disease Control (CDC) has grouped such subjects not infected with the human immunodeficiency virus (HIV) in a special category with specific guidelines for physicians in the USA [4]. In general, screening for tuberculosis and hepatitis $B$ with vaccination for influenza, pneumococcus, and hepatitis $\mathrm{A} / \mathrm{B}$ is recommended by most societies prior to initiating therapy with biologic agents. What Dr. Pham and colleagues indicate is that common immunizations and screening tests are suboptimally utilized in the IBD population prior to commencing long-term biologic therapy. In fact, influenza vaccine was administered in only $43 \%$ and pneumococcal vaccine in mere $24 \%$ of the studied cases. Only $31 \%$ of the patients were serologically tested or received hepatitis A vaccine. Interestingly, the likelihood of appropriate pneumococcal vaccination substantially increased in older patients-possibly a reflection of their age or associated non-IBD-related comorbidities, such as cardiovascular, pulmonary or endocrinologic disease rather than their treatment status. This data seem to parallel earlier national reports of immunization underutilization, as rates of effective vaccination of the general adult public in the USA remain low, despite widespread adaptation of numerous vaccination protocols. In its morbidity and mortality weekly report published in 2015 and latest surveillance data from 2012 influenza season, the CDC have reported only marginal vaccination rates for common pneumococcal ( $21 \%$ in high-risk to $60 \%$ in adults $>65$ years), tetanus (56-64\% in all adults), hepatitis A (16\% in travelers at risk and $14 \%$ in patients with chronic liver conditions), hepatitis 
B (14-26\% in diabetic patients, $34 \%$ in patients with chronic liver conditions, and $62 \%$ in healthcare personnel), Herpes zoster $(24 \%$ in adults aged $>60)$, human papilloma virus $(7.7 \%$ for males aged $19-21$ and $37 \%$ in women aged $19-26$ years), and influenza (38\% in adults, $67 \%$ in healthcare personnel)—substantially below the established goals of $70 \%$ and $90 \%$ for vaccinations in adults and in healthcare workers, respectively $[5,6]$. This outcome was considerably lower in IBD patients who are at risk of developing vaccine-preventable illnesses. Actually, a recent analysis of patient reports indicated that $24 \%$ of IBD patients were vaccinated for influenza, with an $8 \%$ rate for pneumococcal vaccination, varicella $(7 \%)$, hepatitis B (14\%), and tetanus (45\%), while as many as half of the IBD patients may not have been aware of the need for certain vaccinations [7], certainly a worrisome trend in this special population group.

Offsetting patient-perceived barriers, including the cost of vaccination and obliviousness to physician's advice [8] requires additional focus from policymakers in an attempt to improve the overall effective immunization rate by educating patients and medical providers. Dr. Pham and colleagues point out that automated computer reminder to vaccinate patients treated with infliximab translated into higher rates of adherence to established guidelines. Indeed, such patients treated with infliximab were significantly more likely than patients receiving adalimumab to receive pneumococcal vaccine or undergo screening for hepatitis $\mathrm{A} / \mathrm{B}$ and tuberculosis. The rapid development and widespread implementation of computer technology in today's medical practice may ultimately enhance preventative practices in the IBD population. Indeed, studies in Europe have shown major improvement in adherence to vaccination guidelines among gastroenterology specialists who have adapted a frequent reminder system [8]. Establishing simplified and easy accessible national guidelines focusing on the IBD subgroup, mailing initial and appropriate subsequent electronic reminders to patients and providers, utilization of electronic devices and pop-up checklists would significantly improve preventive practices and decrease infectious complications among Crohn's disease and ulcerative colitis patients in the USA at low cost.

Vaccination practices may vary among community and tertiary care center providers, as well as between primary care physicians (PCP) and subspecialists. Large IBD centers are better equipped in tailoring their practice to nuances of IBD patient care, whereas local clinics may not be as familiar with collateral aspects of the disease, including preventative care. Gastroenterologists, who composed $70 \%$ of the physicians in Dr. Pham's study, may typically defer initial screening and vaccine inoculation to PCPs, who in turn, may have the necessary means of administering immunizations but are not comfortable solely managing complex IBD patients. A recent nationwide survey among PCPs in the USA showed that less than two-thirds of the surveyed physicians believe that PCPs were responsible for determining the immunization history [9]. A tendency to under-vaccinate has also been noted across other specialties involved in prescribing immunosuppressive medications, including dermatologists and rheumatologists. Recognition of the high likelihood of future immunosuppression in IBD patients should lead to prompt vaccination against common preventable illnesses at the time of initial diagnosis and certainly prior to initiation of advanced therapy, practice acknowledged by the European Crohn's and Colitis Organization (ECCO) and the American College of Gastroenterology (ACG) $[2,10]$, but has yet to achieve a national scope. With incidence and prevalence in the USA similar to HIV disease, IBD may eventually be assigned a distinct category in the annual recommended adult immunization schedule distributed by the CDC.

In summary, Dr. Pham and colleagues discuss important preventative care concepts relevant to the IBD population, analyzing low rates of vaccination in patients at risk of immunosuppression. They join a long list of clinicians who highlight the observation that many physicians may have decreased awareness of the need to vaccinate, are unsure of the list of necessary immunizations, have concern about the safety and efficacy of inoculations, and have misconceptions about their involvement in the actual administration of vaccinations. Their experience adds to the growing number of calls to enable development of a widely available approach to managing IBD patients at risk of vaccinepreventable illnesses.

Immunization programs have come a long way since Catherine the Great of Russia (who was the first in her nation to get inoculated against smallpox) famously declared on the death of Louis XV of France "it is shameful for the King of France in the eighteenth century to die from smallpox; it is rather barbaric" [11]. Indeed, vaccinations, with among the lowest cost/benefit of all medical treatments, have become an integral tool in averting many potential complications in already sick patients. Their use belongs in the everyday practice of all practitioners.

\section{Compliance with ethical standards}

Conflict of interest None.

\section{References}

1. CDC. https://www.cdc.gov/ibd/pdf/inflammatory-bowel-diseasean-expensive-disease.pdf. 2017. Accessed on August 20, 2017.

2. Rahier JF, Ben-Horin S, Chowers Y, et al. European evidencebased consensus on the prevention, diagnosis and management of 
opportunistic infection in inflammatory bowel disease. J Crohns Colitis. 2009;3:47-91.

3. Pham HV, Hasan I, Udaltsova N, et al. Rates and predictors of vaccinations among inflammatory bowel disease patients receiving anti-tumor necrosis factor agents. Dig Dis Sci. (Epub ahead of print). https://doi.org/10.1007/s10620-017-4716-6.

4. Kim DK, Riley LE, Harriman KH, et al. Recommended immunization schedule for adults aged 19 years or older, United States, 2017. Ann Intern Med. 2017;166:209-219.

5. Williams WW, Lu PJ, O'Halloran A, et al. Centers for Disease Control and Prevention (CDC). Vaccination coverage among adults, excluding influenza vaccination-United States, 2013. MMWR Morb Mortal Wkly Rep. 2015;64:95-102.

6. Lu PJ, Santibanez TA, Williams WW, et al. Surveillance of influenza vaccination coverage-United States, 2007-08 through 2011-12 influenza seasons. MMWR Surveill Summ. 2013;62:1-28.
7. Melmed GY, Ippoliti AF, Papadakis KA, et al. Patients with inflammatory bowel disease are at risk for vaccine-preventable illnesses. Am J Gastroenterol. 2006;101:1834-1840.

8. Christensen KR, Steenholdt C, Buhl SS, et al. Systematic information to health care professionals about vaccination guidelines improves adherence in patients with inflammatory bowel disease in anti-TNFa therapy. Am J Gastroenterol. 2015;110:1526-1532.

9. Gurvits GE, Lan G, Tan A, et al. Vaccination practices in patients with inflammatory bowel disease among general internal medicine physicians in the USA. Postgrad Med J. 2017;93:333-337.

10. Farraye FA, Melmed GY, Lichtenstein GR, et al. ACG clinical guideline: preventive care in inflammatory bowel disease. Am J Gastroenterol. 2017;112:241-258.

11. Pchelov YV. Romanovy. Istoriya dinastii. Moscow: Olma-Press; 2003:120. 